\title{
Modelos de simulación urbana: Experiencia a partir de un juego de estrategia*
}

\author{
Urban simulation models: \\ Experience from a game of strategy
}

Javier Ruiz-Tagle V.** + Alberto Gurovich***

\section{$<$ Resumen>}

Esta experiencia considera el logro de un curso para arquitectura y geografía. La investigación penetra en una experimentación basada en la teoría y dinamica de los sistemas, que ayudan a entender la estructura y la dinámica de la ciudad como sistema complejo. Simcity, un juego de la estrategia que permite el diseño, el planeamiento, y el manejo de la dinámica de la ciudad, se utiliza como software. La experiencia tenía como objetivo principal abordadar las fórmulas de la experimentación didáctica, que integran, mediante el uso de las redes y de los sistemas, las relaciones complejas que configura la ciudad.

La metodología tenía etapas teóricas y experimentales, además de los logros de un ejercicio de simulación. La experiencia tienen una recepcion positiva, como método para aprender y para la investigación, a pesar de que ser extraña en un principio. También, es capaz de generar buenas preguntas de investigación, puesto que el comportamiento de muchas variables fue aprendido para ser visualizado simultáneamente.

$<$ Abstract>

This experience considers the achievement of a course for architecture and geography. The research penetrates into an experimentation based on (the) Systems Theory and (the) Systems Dynamics, which helps understand the structure and the dynamics of the city, as a set of complex systems.

Simcity, a game of strategy that allows the design, planning, and management of the dynamics of a city, is used as software. The experience had as a principal target tackling formulae of didactic experimentation, which integrate, by the application of networks and systems, the complex relations that configures the city.

The methodology had theoretical and experimental stages, plus the achievement of a simulation exercise. The experience had a very good reception, as a method for learning and for research, despite the fact that it seemed strange in the beginning. Also, it generated a increased aptitude for the realization of good research questions, since the behavior of many variables were learnt to be visualized simultaneously.

<PALABRAS CLAVE>

TEORÍA DEL SISTEMA / DINÁMICA DEL SISTEMA / MODELOS / SIMULACIÓN / EXPERIMENTACIÓN

<KEY WORDS >

SYSTEM THEORY / SYSTEM DYNAMICS / MODELS / SIMULATION / EXPERIMENTATION

\section{Introducción}

Preguntarse acerca de lo que significan los modelos para los estudios urbanos, es inevitablemente hacer una reflexión crítica acerca de la condición y la situación científica de la propia disciplina. Ante esto, siempre es bueno señalar que la ciencia no es más que asumir un proceso de adquisición y refinado del conocimiento de manera rigurosa. Si bien históricamente ha habido detractores de la ciencia, de los cuales uno de los últimos

El texto resume la investigación del proyecto Mecesup UCH-0217, para Experiencias Docentes 2005-2006, realizado por los dos autores.

** Arquitecto y Magíster en Urbanismo, Universidad de Chile. Ayudante del Departamento de Urbanismo, FAU.

*** Arquitecto, Profesor Asociado del Departamento de Urbanismo, FAU. 
fue la crítica de la postmodernidad, toda investigación, independiente de su ideología, puede ser ciencia.

En términos de su desarrollo, las ciencias establecen grados de madurez por cuanto asumen ascendentemente caracteres de exploratorias, descriptivas, correlacionales, y finalmente, explicativas. Si observamos e estado del arte del urbanismo en Chile, se podría decir que está a un nivel descriptivo, ya que solo se están especificando las propiedades de los fenómenos urbanos. En los países industrializados, en cambio, los estudios urbanos podrían estar a un nivel correlacional. Respecto de esta situación, es posible señalar dos problemas importantes con respecto al estado y al avance de la disciplina urbanística, dentro del marco de las ciencias sociales y humanas. El primer problema, tiene que ver con el fenómeno de la 'reflexividad', en donde, al investigar y exponer un fenómeno, se afecta el desarrollo y transformación del fenómeno mismo. En el caso del urbanismo, por ejemplo, si se otorga mucha difusión científica de una determinada visión sobre un problema, se corre el riesgo de afectar las formas de operación de los actores del desarrollo urbano.

Un segundo problema, hace alusión a una bella metáfora de la historia de la filosofía; el búho de Minerva, aquel que, como señalaba Hegel en la Filosofía del Derecho, despliega sus alas al anochecer. "La teoría y la filosofía simbolizadas en el búho de Minerva, siempre llegan tarde; sólo se constituyen como conocimiento una vez que la vida real de las sociedades produjo los acontecimientos y las circunstancias que motivan la reflexión del teórico y del filósofo» ${ }^{1}$.

Asumiendo estas falencias, y entendiendo el urbanismo como una disciplina esencialmente proyectual, alimentada de lo social y lo político, es que surge la intención de reorientar nuestros esfuerzos. En el sentido de lo que Marx plantea, la misión de la ciencia, no puede extinguirse en la sola contemplación del mundo, sino por el contrario, su deber es transformarlo. Desde este punto de vista, es que visualizamos la importancia de asumir la investigación urbana como ciencia. En el urbanismo no se está enseñando a enfrentar problemas complejos de manera rigurosa, y se prefieren las simplificaciones. En el mismo sentido, hay resistencia para incorporar el paradigma científico a las disciplinas, en virtud de generar nuevo conocimiento. Por suerte, los médicos si lo han hecho. Por mala suerte, los urbanistas no hemos hecho lo suficiente.

Ahora bien, en virtud de ser una disciplina propositiva, tenemos la noción de que no en todas las ciencias se puede experimentar. En el urbanismo no ha habido predicción de procesos, ya que no se puede investigar experimentando. Entonces, ¿Qué pasa si se estudian las posibles intervenciones del futuro, tomando en cuenta que el urbanismo es una disciplina esencialmente proyectual? ¿No es parte de la investigación científica, estudiar las soluciones a los problemas planteados? ¿Por qué en el urbanismo sólo se investigan los problemas y no las soluciones? El problema sin duda está en el objeto de estudio. ¿Es posible experimentar posibles 'curas' para la ciudad, tal como hacen en las ciencias duras con las ratas de laboratorio? La respuesta, a simple es vista, es un NO rotundo.

A pesar de esto, podemos encontrar respuestas en el desarrollo de otras disciplinas. En la economía por ejemplo, se proponen nuevos modelos económicos, a pesar de que la experiencia plantea que es muy delicado ponerlos a prueba sin estar seguros de su posible éxito. De este modo, el desarrollo de las matemáticas, de la estadística y de las tecnologías de información, ha dado espacio a la experimentación de posibles condiciones futuras, en términos de poder introducir modificaciones y observar los posibles efectos al sistema general. Son éstas, las técnicas de modelamiento que recién estamos aprendiendo a utilizar para los estudios urbanos. En el largo camino que se pretende iniciar, la experiencia que señala este texto, busca relevar la importancia de acercar el urbanismo, a un estado de ciencia explicativa.

\section{Магсо †ео́rico}

Teoría de Sistemas:

Un paradigma alternativo para

el estudio de la ciudad

El origen de la Teoría de Sistemas, dice relación con la aparición del método analítico, y en general, del positivismo. Se aspira a una homologación de las 'ciencias duras', de manera diferente a la dialéctica y la hermenéutica. De esta manera, se buscan propiedades comunes de las entidades (o sistemas) en todos los niveles, tratando de conformarse como una 'metateoría'. Para ello, establece cuatro pasos básicos: reducir, comprender, modelar y predecir. En términos sintéticos, el objeto de estudio de la TS son los sistemas complejos, su método es el estudio analítico del comportamiento esencial, y su objetivo es facilitar el desarrollo teórico en campos de difícil abstracción del objeto. Uno de los 'campos de difícil abstracción' más cercano que tenemos, es el urbanismo y la ciudad. Sin embargo, pocos estudios han enfrentado su temática desde la TS.

Dentro del campo de esta teoría, un concepto clave a analizar es la autopoiesis ${ }^{2}$, o propiedad de un ente de mantener una estructura estable. Al mantenerse estable en una situación dinámica, se convierte en un sistema.

En ciencias sociales, a partir de las elaboraciones del alemán Luhmann ${ }^{3}$, la TS aporta en el estudio de una complejidad siempre creciente en las sociedades, generándose constantemente nuevos sistemas. Los sistemas sociales en particular, se definen como autorreferentes, esto es, sólo se comunican si se necesitan, en una situación llamada 'doble contingencia'. Se deja la dicotomía entre el todo y las partes, para pasar a una relación dicotómica entre los sistemas y su entorno.A su vez, estos sistemas se caracterizan por una diferenciación y una especialización progresiva, en un particular espiral de complejidad: diferenciación, especialización, fragmentación y posterior aumento de la complejidad.

\section{Dinámica de Sistemas y modelos de ciudad}

La Dinámica de Sistemas surge como un desarrollo posterior de la TS. En términos sintéticos, la DS emana de la ingeniería industrial, su objeto de estudio es la evolución e interacción de los sistemas en el tiempo, su método es el modelamiento (incorporando análisis y síntesis), y su objetivo es entender sistemas organizacionales complejos, para tomar decisiones más efectivas. Se entiende a las organizaciones acá, como sistemas sociales complejos, dinámicos y no lineales, en contraposición al análisis clásico de lo estático, local y lineal. Si antes se pretendía pronosticar y optimizar el futuro, la DS pretende estudiar múltiples futuros posibles con respecto a diferentes estrategias. 
La organización y planificación de los sistemas dinámicos no puede estar basada únicamente en el pasado. Se deben descubrir estructuras causales en los sistemas, es decir, sus lógicas de comportamiento, de manera de relacionar las decisiones con las consecuencias. De esta manera, la DS plantea más allá de predecir, el hecho de revelar los supuestos ocultos ante interpretaciones obvias o socialmente asumidas. A partir de la idea de que un modelo es una idealización de una realidad, utilizada para plantear y analizar la naturaleza de un problema de manera simplificada, el modelamiento operativo dentro de la perspectiva de la DS, representa un laboratorio libre de riesgos, expresado en diagramas de causalidad, definiendo los límites de cada sistema, e incorporando variables exógenas y endógenas.

¿Puede la ciudad ser entendida como un sistema dinámico? Si se la entiende como una suma de elementos o entidades (estáticos y móviles, físicos y sociales) en interacción, que tienen a su vez distintos modos de comportamiento, en un espacio determinado, estamos ante la presencia de un sistema dinámico, siempre que consideremos irrelevantes algunos aspectos.

¿Qué aporte significa estudiar la ciudad como modelo sistémico dinámico? De partida, enfrentar la visión sectorial que se tiene de las partes de la disciplina. El sistema de transporte, el sector vivienda, las áreas verdes, la infraestructura, los servicios, etc. El enfoque sistémico, siempre con la ayuda de herramientas tecnológicas de resolución de complejidades, permite analizar tanto la totalidad de la ciudad como gran sistema, y pequeños sectores como subsistemas no aislados.

\section{Antecedentes de la experiencia de investigación}

La experiencia considera la realización de un curso de pregrado para arquitectura y geografía, en paralelo con una investigación entre docentes y estudiantes. Para esto, se utilizan técnicas de aprendizaje y simulación de sistemas urbanos, interpretando su desarrollo, dirección y control. En ello se utiliza un software configurado como juego de estrategia, denominado SimCity. Los modelos que implica, en cuanto contienen datos, variables, y expresiones tridimensionales, permiten aislar factores y representar estrategias de acción en el encuadre de una variedad de escenarios posibles, combinando técnicas habituales en el uso de sistemas de información geográfica, herramientas normativas, de gestión, de planificación y de diseño urbano.

En términos de programación, SimCity es un juego configurado en base a 'agentes'. Éstos pueden ser elementos del paisaje, unidades de vivienda, unidades de comercio, unidades de industria, infraestructura de transporte, infraestructura de energía y saneamiento, y equipamiento cívico. La característica principal de los 'agentes', es que poseen un comportamiento lógico propio ante otros estímulos y/o ante otros agentes, lo que se traduce ciertamente en un sistema, según el marco teórico anteriormente expuesto. De esta manera, todos estos elementos interactúan en un gran sistema, generando diferentes desarrollos ante diferentes decisiones de diseño y planificación urbana, y la forma de administrarlas. A su vez, la interfase del programa dispone de una serie de indicadores que van dando cuenta de la situación de la ciudad creada.

Los indicadores urbanos más útiles, los podemos clasificar en cuatro grupos:

1. Medición de demanda de residencia, comercio, e industria.

2. Cartas de información geográfica: muestran zonificaciones de peligro de incendio, crimen, cobertura de educación, demanda habitacional, cobertura de agua potable, cobertura de electricidad, estado del tráfico, zonas por uso de suelo, valor del suelo, 'rating' del alcalde, edad de habitantes, cobertura de salud, contaminación del aire, contaminación del agua, recolección y tratamiento de basuras.

3. Gráficos de comportamiento en el tiempo de: muestran valores de crimen, suministro de energía, suministro de agua, contaminación del aire, empleo y población, contaminación del agua, recolección de basuras, cobertura de educación, educación por edad, población por edad, esperanza de vida, ingreso per cápita, ingresos v/s egresos municipales, fondos municipales, demanda RC (residencia, comercio e industria), 'rating' del alcalde, volumen de tráfico.

4. Presupuesto mensual; ingresos y egresos.

\section{Objetivos}

Se pretende como objetivo abordar fórmulas de experimentación didáctica, que integren, mediante la aplicación de redes y sistemas, las relaciones organizacionales complejas que sustentan y dinamizan las formaciones territoriales. Asimismo, identificar las causas, los principios teóricos, las motivaciones ideológicas y los demás estímulos que propulsan, configuran y articulan los elementos de composición socioespacial de los territorios urbanizados. Finalmente, participar en la ejercitación de métodos de intervención urbanística, cuyos procedimientos puedan ser contrastados con el accionar contemporáneo.

\section{Metodología}

La metodología del curso, consideró tres etapas teóricas: i) la ciudad como sistema dinámico, ii) sistemas y subsistemas; construcciones ideológicas de los modelos, y iii) estrategias de diseño, gestión y planificación urbana. A su vez, se realizaron tres etapas experimentales: i) introducción a SimCity, experimentación y descubrimiento de modelos, ii) trabajo en subsistemas, y iii) modelamiento y simulación de problemas urbanos.

En términos de generación de productos de investigación, se encargó un primer trabajo en el cual se trabaje un problema de investigación y se modelen sus relaciones estructurales en función de dilucidar puntos críticos de posible intervención y/o simulación. En segunda instancia, se encargó tomar el problema de investigación del primer trabajo y modelar sus relaciones estructurales en la plataforma SimCity, en función de visualizar posibles consecuencias ante diversas intervenciones de diseño urbano. Más que llegar a la mejor solución, lo que interesaba en este caso era la investigación en torno al proceso de toma de decisiones, los efectos que acarrea cada una, la comprensión multicausal de los procesos urbanos, y las conclusiones que pueden emanar para alimentar la teoría. Para documentar dichos procesos, cada clase se registraban las situaciones iniciales, los pasos de intervención y las situaciones finales, mediante los indicadores que posee el software y las imágenes de la configuración urbana que adquiría el modelo de ciudad creada.

\section{Exposición de trabajos}

\section{Miguel Aguilera, estudiante de arquitectura}

- Objetivo: Poner a prueba la localización de equipamientos de salud y educación en dos zonas de una ciudad, a través del manejo de los radios de atención de cada centro, de manera de registrar cómo afectan el valor de suelo, la demanda de residencia y el acceso a estos servicios. 

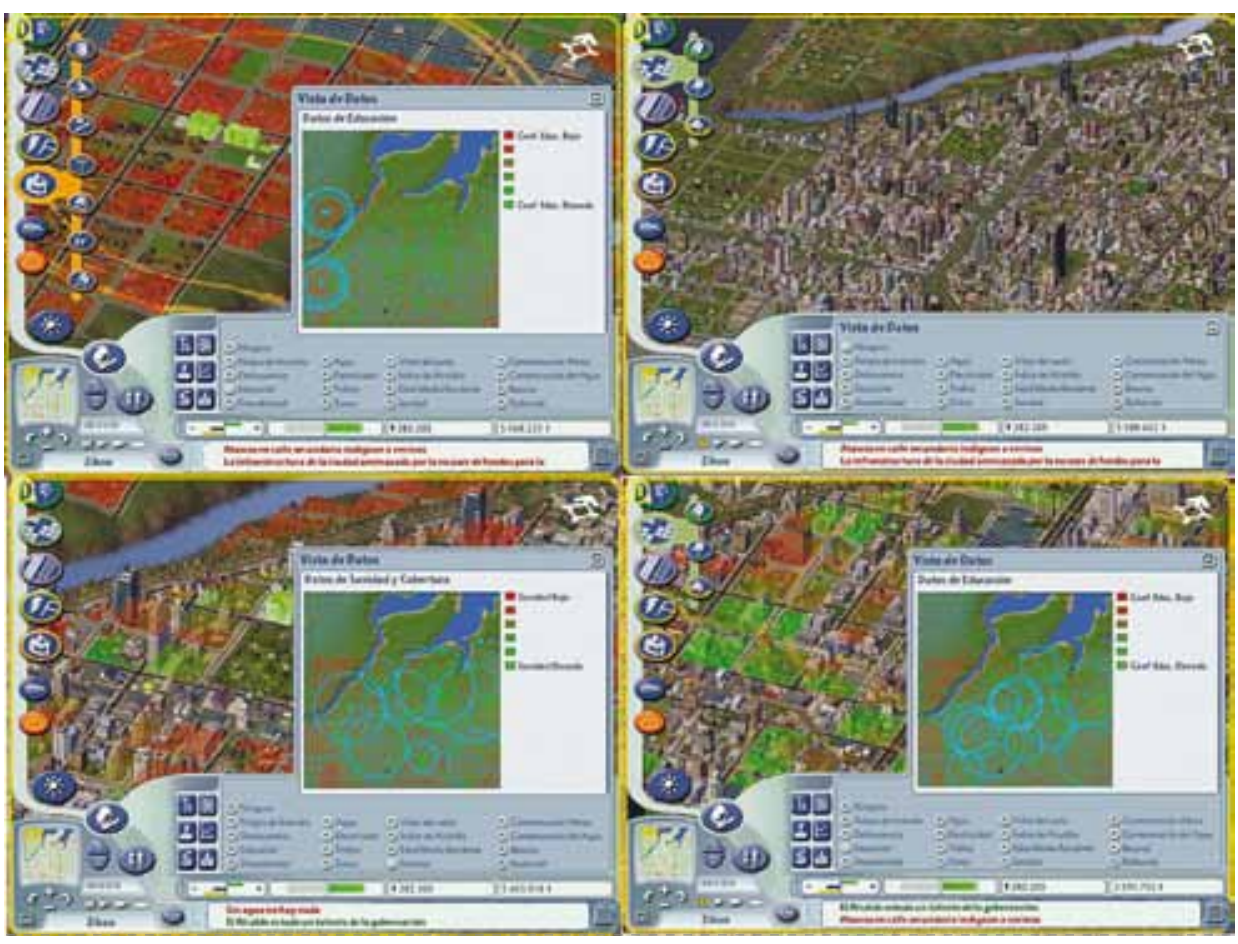

- Situación inicial: Ciudad de 200.000 habitantes, con equipamientos de salud y educación distribuidos homogéneamente.

- Intervención 1: Reorganización de los centros de salud en función de zonas de alta densidad.

- Efecto 1: Reorganización de la ocupación residencial. Se despejan sus principales variables; radios de acción y capacidad de atención.

- Intervención 2: Eliminación de cobertura de salud y educación por completo.

- Efecto 2: Abandono lento y generalizado de la ciudad.

- Intervención 3: Redistribución y concentración de equipamientos en dos zonas que antes no tenían buena cobertura.

- Situación final: Valor de suelo no sufre cambio drástico. Fuerte desarrollo del comercio y vivienda de alto estándar en zonas bien equipadas. Abandono residencial en zonas no equipadas (Figura $1)$.
Sebastián Vivero, estudiante de geografía

- Objetivo: Experimentar acerca de mecanismos para mejorar los niveles de contaminación de una ciudad, a través del tipo de industria y el manejo de áreas verdes.

- Situación inicial: Ciudad densa con baja contaminación. contaminación mediante intervenciones (cambio del uso del suelo, disminución de impuestos a industrias contaminantes y instalación de planta energéticas contaminantes).

- Efecto 1: Disminución de población.

- Intervención 2: Alza de impuestos a industrias contaminantes y baja de impuestos a industria limpia.

- Efecto 2: Reconversión industrial, baja de contaminación, pero persisten focos.

- Intervención 3: Incorporación de biomasa en forma de plantación de árboles.

- Efecto 3: No bajó contaminación. Juego no considera efecto ecológico de árboles.
- Intervención 1: Aumento de la
Figura 1

- Intervención 4: Incorporación de áreas verdes recreacionales (plazas y parques)

- Efecto 4: Reducción de contaminación cercana, mejora de los focos.

- Situación final: La ciudad mejoró sus índices de contaminación ambiental. Sin embargo, el resultado final provocó aumentos de cesantía, aumento en los gastos del municipio, cambios en el uso y valor del suelo, etc. (Figura 2).

\section{Arturo Palma}

\section{estudiante de arquitectura}

- Objetivo: Poner a prueba el sustento del desarrollo de un polo urbano aislado, a través de los sistemas de transporte.

- Situación inicial: Un sector de ciudad sin residencias, con actividad laboral y de servicios. Otro sector aislado predominantemente residencial, con algunos servicios.

- Intervención 1: Creación de focos de desarrollo en la periferia del paño urbanizable, sumada a una red de metro que los vincule. Fundamentalmente industria, para satisfacer demanda de empleos y bienes. 
- Efecto 1: El núcleo industrial creado no afecta ni la contaminación, ni el valor de suelo.

- Intervención 2: Definición de una estación periférica y una estación céntrica.

- Efecto 2: Uso del metro bastante bajo, e ineficiente. Producía pérdidas al presupuesto de la ciudad. Uso del metro se concentraba sólo en zonas densas de la ciudad.

- Intervención 3: Potenciamiento de la estación céntrica, e inserción de un núcleo comercial cerca de la estación periférica.

- Efecto 3: Subió porcentaje de uso del metro.

- Situación final: Los polos periféricos se desarrollaron a ritmo lento. Para potenciar la actividad del metro, fue necesario generar polos comerciales densos en cada estación. El crear un polo de desarrollo industrial alejado de la ciudad, permitió no bajar el valor de suelo de las áreas más demandadas y satisfacer las necesidades de trabajo y bienes de consumo de la zona periférica. La conectividad del metro fue un factor primordial en el desarrollo conjunto de la ciudad y la industria, ya que era el único medio por el cual estaban unidos (Figura 3).

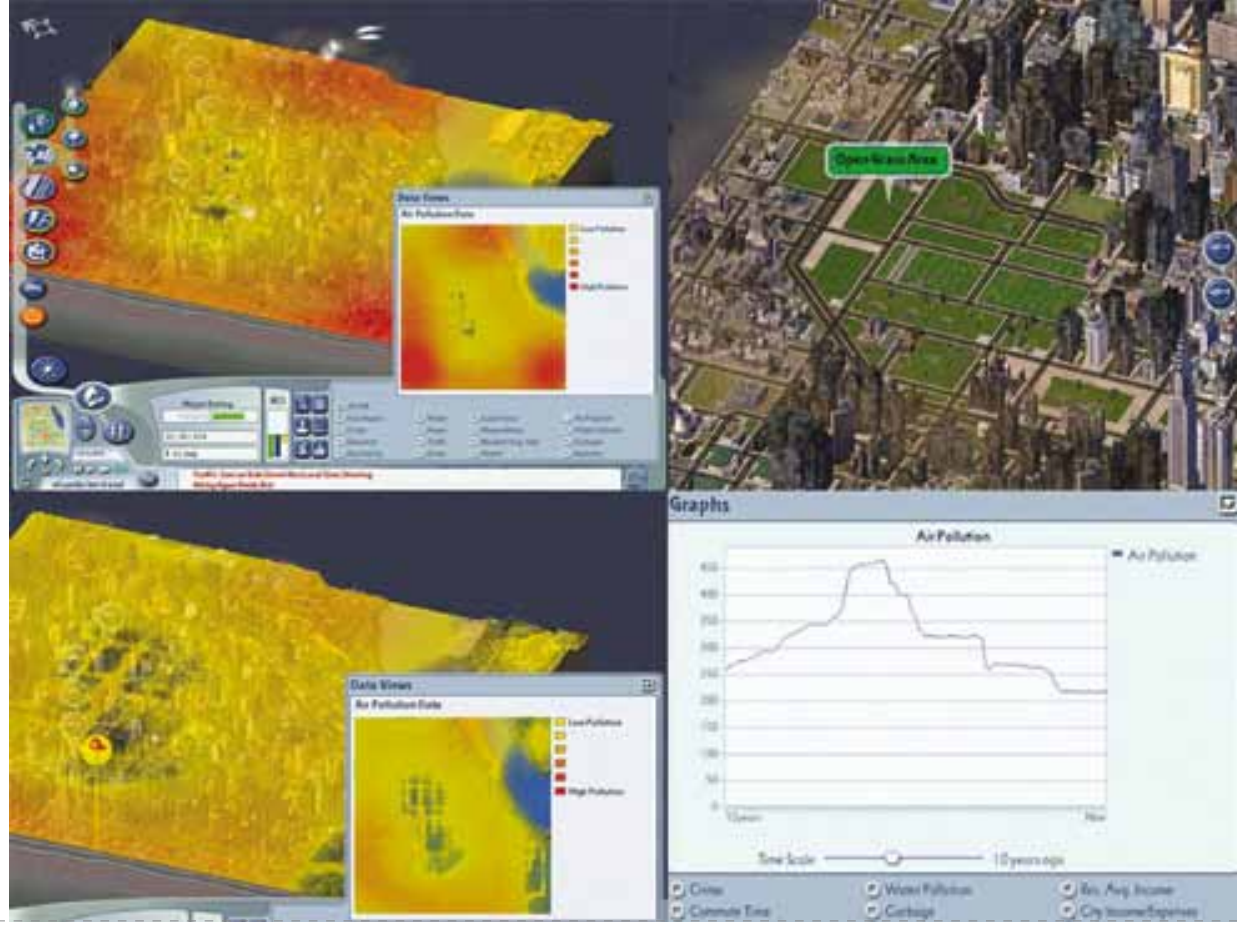

\section{Conclusiones}

La realización del curso, si bien tuvo inconvenientes de gestión administrativa que redundaron en la planificación de los tiempos de exposición teórico y experimental, tuvo una muy buena acogida por parte de los estudiantes, como método de aprendizaje, de investigación y por la interdisciplinariedad del planteamiento.

La experiencia sostiene el incentivo particular que representa el enseñar y aprender modelos de simulación sistémicodinámicos a partir de un juego de estrategia. En un principio, parecía extraño enfrentar la dinámica urbana con un juego, pero luego, la complejidad y la elaboración conceptual en torno a éste, resultó muy atractiva y novedosa. La experimentación con modelos causales y con el juego, generó en los estudiantes una importante capacidad de realizar buenas preguntas de investigación, ya que expone fácilmente todas las variables sobre un problema y sus relaciones estructurales.

Se aprendió a simular problemas reales, y en el ejercicio de hacer que funcionen, se pudo descubrir las razones de la ocurrencia de éstos. Los ejercicios se entendieron como trabajos prácticos, que lograron entregar más contenido que un trabajo teórico. Se logró tener, paradojalmente, una apreciación más real de los problemas de la ciudad, y de cómo las diferentes variables influyen en su funcionamiento.

Finalmente, se puede destacar la importancia de contar con nuevas tecnologías con una gran capacidad de manejo de datos, para la experimentación, a modo de laboratorio, de escenarios futuros para la ciudad.

\section{Bibliografía}

Amendola, G. La Ciudad Postmoderna, magia y miedo en la metrópolis contemporánea. Madrid: Celeste Ediciones, 2000.

Borón, A. Tras el búho de Minerva. Mercado contra democracia, en el capitalismo de fin de siglo. Argentina: Fondo de Cultura Económica, 2000.

Bunge, M. La investigación científica: Su estrategia y su filosofía. Barcelona: Ariel, 1969.

Ferré, A. et al. Verb, Architecture Boogazine: Connection. Barcelona: Actar, 2004.

Forrester, J.W. Urban dynamics. Cambridge, Massachusetts: Institute of Technology, 1969.

Freire, J. «SimCity y la planificación urbana». Reflexiones personales e información sobre la sociedad y el conocimiento abiertos, 15 abril 2005. En: http://nomada.blogs.com/ jfreire/2005/04/simcity_y_la_pl.html 

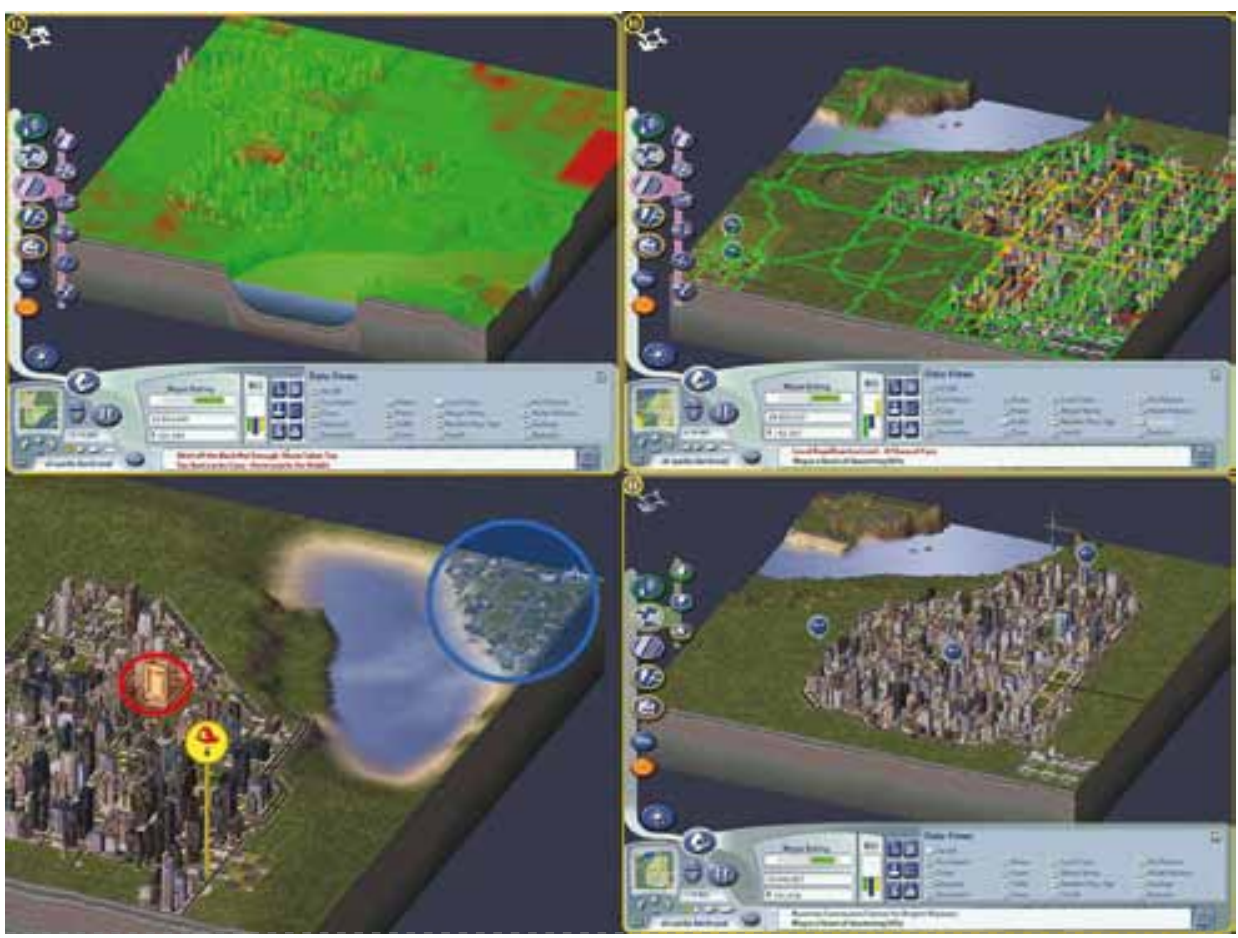

Friedman, T. "The Semiotics of SimCity». En: First Monday, peer-reviewed journal on the internet. En: http://www.firstmonday.org

Gurovich, A.; Ruiz-Tagle, J.. «Modelos de simulación operativa, aplicados en planificación y diseño urbano». Programa oficial del curso de Urbanismo Avanzado I. Santiago, Chile: Facultad de Arquitectura y Urbanismo, Universidad de Chile, 2006.

Instituto Tecnológico de Sonora. Dinámica de Sistemas. En: www.itson.mx

Johnson, S. «Emergence: The Connected Lives of Ants, Brains, Cities, and Software». En el artículo 'Sim City' de Ferré, A. et al. Verb, Architecture Boogazine: Connection. Barcelona: Actar, 2004

Lobo, D. «A city is not a toy: How SimCity Plays with Urbanism». London School of Economics and Political Science, Cities Programme: Architecture and Engineering. Discussion Paper Series, Londres, 2004.

López, L.; Zúñiga, R. Dinámica de Sistemas y la nueva tecnología para la toma de decisiones complejas: Mapeo y simulación organizacional. En página web: www.oit.or.cr/ mdtsanjo/actemp/ilgo2002/PresRoyZuniga 1.pdf
Luhmann, N. Introducción a la teoría de sistemas. México D.F.: Universidad Iberoamericana, 1996

Martínez, F.; Donoso, P. «MUSSA: Un modelo de equilibrio del uso del suelo con externalidades de localización, planos reguladores y políticas de precios óptimos». Ponencia Universidad de Chile, $X$ Congreso Chileno de Ingeniería de Transporte. Concepción, 2001.

Maturana, H.; Varela, F. De máquinas y seres vivos. Autopoiesis: la organización de lo vivo. Buenos Aires: Universitaria Lumen, 2004.

Ruiz-Tagle, J. «Urbanismo y Simulación. Simcity entre la cognición y la planificación de la ciudad». Trabajo final Taller de Urbanismo $2^{\circ}$ Semestre, Magíster en Urbanismo. Universidad de Chile, 2005. 